\title{
Natural and dynamical neutrino mass mechanism at the LHC
}

\author{
Julia Gehrlein, ${ }^{1,2, *}$ Dorival Gonçalves, ${ }^{3, \dagger}$ Pedro A. N. Machado, ${ }^{4, \ddagger}$ and Yuber F. Perez-Gonzalez ${ }^{5,6, \S}$ \\ ${ }^{1}$ Departamento de Física Teórica, Universidad Autónoma de Madrid, \\ Cantoblanco, E-28049 Madrid, Spain \\ ${ }^{2}$ Instituto de Física Teórica UAM/CSIC, Calle Nicolás Cabrera 13-15, \\ Cantoblanco, E-28049 Madrid, Spain \\ ${ }^{3}$ Department of Physics and Astronomy, University of Pittsburgh, \\ 3941 O'Hara Street, Pittsburgh, Pennsylvania 15260, USA \\ ${ }^{4}$ Fermi National Accelerator Laboratory, Batavia, Illinois 60510, USA \\ ${ }^{5}$ Departamento de Física Matemática, Instituto de Física, Universidade de São Paulo, \\ Rua do Matão 1371, CEP. 05508-090 São Paulo, Brazil \\ ${ }^{6}$ ICTP South American Institute for Fundamental Research \& Instituto de Física Teórica, UNESP, \\ Rua Dr. Bento T. Ferraz, 271, CEP. 01140-070 São Paulo, Brazil
}

(Received 10 May 2018; revised manuscript received 4 July 2018; published 30 August 2018)

\begin{abstract}
We generalize the scalar triplet neutrino mass model, the type II seesaw. Our model displays compelling theoretical and experimental features. First, lepton number violation is dynamically generated, without introducing a naturalness problem, nor relying on arbitrarily small parameters. Second, we identify a smoking gun signature at the LHC that both probes the triplet structure of our seesaw mechanism and allows to disentangle it from the usual type II scenario. Additionally, we discuss other interesting phenomenological aspects of the model such as the presence of a massless Goldstone boson and deviations of standard model Higgs couplings.
\end{abstract}

DOI: $10.1103 /$ PhysRevD.98.035045

\section{INTRODUCTION}

The presence of nonzero neutrino masses, as inferred by neutrino oscillation experiments, is the only laboratorybased evidence of physics beyond the standard model [1,2]. Strictly speaking, neutrinos have no mass in the standard model (SM). There is no unique prescription of how neutrinos could become massive. Perhaps the simplest way of generating neutrino masses is via the seesaw framework. In its naïve realizations, seesaw types I, II and III [3-10], a large suppression of the electroweak breaking scale provides an explanation for the smallness of neutrino masses. Without a full underlying framework, like grand unified theories or supersymmetry, these mechanisms typically introduce a hierarchy problem due to the large mass gap [11] or rely on very small (but technically natural [12]) parameters.

\footnotetext{
*julia.gehrlein@uam.es

dorival.goncalves@pitt.edu

*yfperezg@if.usp.br

pmachado@fnal.gov
}

Published by the American Physical Society under the terms of the Creative Commons Attribution 4.0 International license. Further distribution of this work must maintain attribution to the author(s) and the published article's title, journal citation, and DOI. Funded by SCOAP ${ }^{3}$.
In general, the seesaw mechanism generates a small parameter from the ratio of two disparate physics scales, e.g., electroweak versus grand unification scales. Therefore, when we set the new heavy states to the weak scale (such as done in studies of type II seesaw at colliders $[13,14])$, the "seesaw" mechanism is exchanged by a small parameter. This can be appreciated in a model independent way by writing down schematically the Weinberg effective operator that generates neutrino masses [15], namely

$$
\mathcal{L}_{5}=\frac{c}{\Lambda} L L H H
$$

( $H$ and $L$ are the Higgs and lepton doublets) and observing that if $\Lambda \sim\langle H\rangle$ then the Wilson coefficient $c$ needs to be tiny in order to obtain sub-electronvolt neutrino masses. We will show in this Letter that a simple generalization of the type II seesaw, replacing the seesaw by a chain of seesaws, can completely avoid the postulation of a new scale. The model is entirely at the weak scale, and a small lepton number breaking is dynamically generated.

More concretely, in type II seesaw a scalar triplet

$$
\Delta=\left(\begin{array}{cc}
\delta^{+} / \sqrt{2} & \delta^{++} \\
\left(v_{\Delta}+\delta+i a_{\delta}\right) / \sqrt{2} & -\delta^{+} / \sqrt{2}
\end{array}\right)
$$

obtains its vacuum expectation value (vev) after electroweak symmetry breaking 


$$
v_{\Delta} \simeq \frac{\mu}{\sqrt{2}} \frac{v^{2}}{M_{\Delta}^{2}},
$$

where $\mu$ is a dimensionful lepton number breaking parameter of the scalar potential, $v=246 \mathrm{GeV}$ is the Higgs doublet vev, and $M_{\Delta}$ is approximately the physical mass of $\Delta$. Neutrino masses are given by $m_{\nu}=\sqrt{2} Y v_{\Delta}$, with $Y$ being a matrix of Yukawa couplings.

We can immediately see that the smallness of neutrino masses can only be obtained by having small Yukawas, large $M_{\Delta}$, and/or small ad hoc lepton number breaking parameter $\mu$. For instance, if $M_{\Delta}$ is accessible at the LHC, say at the $\mathrm{TeV}$ scale, and the Yukawas are taken to be of order 1, we obtain

$$
\mu \simeq 1.6 \mathrm{eV}\left(\frac{m_{\nu}}{0.1 \mathrm{eV}}\right)
$$

Since $\mu=0$ restores a symmetry of the Lagrangian, it is not generated by other couplings due to quantum corrections, thus being technically natural in the t'Hooft sense [12]. Nevertheless, it is unappealing to have this enormous hierarchy of scales $\mu / v \lesssim \mathcal{O}\left(10^{-11}\right)$ put in arbitrarily. As suggested by the considerations made before regarding the Weinberg operator, this is not exclusive to type II seesaw.

In this paper we present a generalization of the type II seesaw scenario which dynamically generates a very low lepton number breaking scale from a small hierarchy. The model is naturally found at the weak scale, introducing no new fine-tuning neither arbitrarily small couplings. Our mechanism engenders a rich phenomenology, including deviations of SM Higgs couplings, presence of a massless Majoron, lepton flavor violation and a smoking gun signature at the LHC that distinguishes this model from the usual type II seesaw.

\section{THE MECHANISM}

The idea simply amounts to replicate the induced vev suppression mechanism with additional scalar singlets, as shown in Fig. 1. In our concrete setup, all mass parameters are near the electroweak scale and all dimensionless couplings are of similar order, thus yielding a natural model of neutrino masses accessible at the LHC. We will focus on a scenario with two extra scalar singlets, as this is the most minimal realization that successfully implements

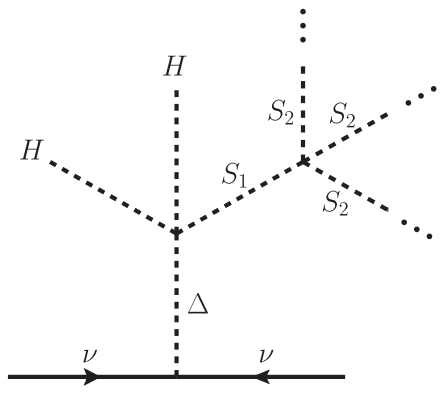

FIG. 1. Illustration of the generalized type II seesaw mechanism for neutrino mass generation.

the mechanism and also exhibits all important phenomenological features of our framework.

First, we require dynamical lepton number breaking. To that end, we promote $U(1)_{\ell}$ lepton number to a global symmetry in which leptons have charge $\ell_{\text {leptons }}=+1 / 2$ (the normalization has been chosen for convenience) and quarks have no charge. The neutrino Yukawa coupling

$$
\mathcal{L}_{\text {Yuk }}^{\nu}=-Y \bar{L}^{c} i \sigma_{2} \Delta L+\text { H.c. }
$$

( $\sigma_{2}$ is the second Pauli matrix, $Y$ is a matrix of Yukawa couplings in flavor space, and ${ }^{c}$ denotes charge conjugation) requires $\ell_{\Delta}=-1$, forbidding the triple coupling $\mu H^{T} i \sigma_{2} \Delta^{\dagger} H$. We introduce the first complex SM singlet scalar $S_{1}$ with lepton number $\ell_{1}=+1$ so its vev may play the role of lepton number violating parameter $\mu$. Then, we generalize the type II seesaw model by invoking another extra scalar singlet with charge $\ell_{2}=1 / 3$, allowing for a term $S_{1}^{*} S_{2}^{3}$ in the scalar potential. All scalars but the Higgs and $S_{2}$ have positive bare mass terms. The crucial point is that when $S_{2}$ develops a vev spontaneously, it induces a suppressed vev for $S_{1}$, which then induces an even smaller vev for $\Delta$. The model can easily be generalized for any number $N$ of scalar singlets, see Appendix A. We identify the usual type II seesaw with a $N=1$-step version of the generalized model in which $S_{1}$ is integrated out. Our model bears similarities with multiple seesaw and clockwork models (see, for instance, Refs. [16-24]).

As we will see later, a simple 2-step realization can lead to small neutrino masses given that some quartic couplings and neutrino Yukawas are of order $10^{-2}-10^{-3}$ (larger couplings can be obtained in realizations with extra steps). Without further ado, we write down the scalar potential

$$
\begin{aligned}
V= & -\frac{m_{H}^{2}}{2} H^{\dagger} H+m_{\Delta}^{2}\left\langle\Delta^{\dagger} \Delta\right\rangle+m_{1}^{2} S_{1}^{*} S_{1}-\frac{m_{2}^{2}}{2} S_{2}^{*} S_{2}+\frac{\lambda_{H}}{4}\left(H^{\dagger} H\right)^{2}+\frac{\lambda_{2}}{4}\left(S_{2}^{*} S_{2}\right)^{2} \\
& +\lambda_{1 H}\left(S_{1}^{*} S_{1}\right)\left(H^{\dagger} H\right)+\lambda_{2 H}\left(S_{2}^{*} S_{2}\right)\left(H^{\dagger} H\right)+\left[\lambda_{A} H^{T} i \sigma_{2} \Delta^{\dagger} H S_{1}^{*}-\frac{2}{3} \lambda_{12}^{\prime} S_{1}^{*} S_{2}^{3}+\text { H.c. }\right] \\
& +\frac{\lambda_{\Delta}}{4}\left\langle\Delta^{\dagger} \Delta\right\rangle^{2}+\frac{\lambda_{\Delta}^{\prime}}{4}\left\langle\Delta^{\dagger} \Delta \Delta^{\dagger} \Delta\right\rangle+\frac{\lambda_{1}}{4}\left(S_{1}^{*} S_{1}\right)^{2}+\lambda_{12}\left(S_{1}^{*} S_{1}\right)\left(S_{2}^{*} S_{2}\right) \\
& \left.+\lambda_{H \Delta}\left(H^{\dagger} H\right)\left\langle\Delta^{\dagger} \Delta\right\rangle+\lambda_{H \Delta^{\prime}}\left\langle H^{\dagger} \Delta \Delta^{\dagger} H\right\rangle+\lambda_{1 \Delta}\left\langle\Delta^{\dagger} \Delta\right\rangle\left(S_{1}^{*} S_{1}\right)+\lambda_{2 \Delta}\left\langle\Delta^{\dagger} \Delta\right\rangle\left(S_{2}^{*} S_{2}\right),\right\} \text { “incidental" terms}
\end{aligned}
$$


where the parameters more relevant for the mechanism and phenomenology are in the first two lines. Although the quartic couplings on third and fourth lines are important for the potential stability, they play almost no role otherwise (thus called "incidental"). The potential stability is not a primary concern of this manuscript, but it is important to note that the quartic couplings $\lambda_{A}$ and $\lambda_{12}^{\prime}$ tend to destabilize the potential, and hence are expected to be small. For more considerations regarding stability see Appendix B. We define the neutral components of the fields as $H^{0}=$ $(v+h+i a) / \sqrt{2}, \quad \Delta^{0}=\left(v_{\Delta}+\delta+i a_{\delta}\right) / \sqrt{2}$ and $S_{j}=$ $\left(v_{j}+s_{j}+i a_{j}\right) / \sqrt{2}$, for $j=1,2$.

The positive mass terms for $\Delta$ and $S_{1}$ ensure that if $\lambda_{A}=$ $\lambda_{12}^{\prime}=0$ then the vevs for these fields are zero. These two quartic couplings are protected from loop corrections by accidental global $U(1)$ symmetries. Moreover, $\lambda_{A}$ and $\lambda_{12}^{\prime}$ can be made real by rephasing the scalar singlet fields. As long as $v_{\Delta}$ and $v_{1}$ are much smaller than $v$ and $v_{2}$, we can obtain the former vevs by treating $H$ and $S_{2}$ as background fields. First, we obtain the approximate vevs of $H$ and $S_{2}$ by setting other scalar fields to zero,

$$
m_{H}^{2}=\frac{1}{2} \lambda_{H} v^{2}+\lambda_{2 H} v_{2}^{2}, \quad m_{2}^{2}=\frac{1}{2} \lambda_{2} v_{2}^{2}+\lambda_{2 H} v^{2} .
$$

Then, by replacing $H$ and $S_{2}$ by their vevs, we can easily calculate the vevs and spectrum of other scalars:

$$
v_{1}=\frac{\lambda_{12}^{\prime} v_{2}^{3}}{3 M_{1}^{2}}, \quad v_{\Delta}=\frac{\lambda_{A} v^{2} v_{1}}{2 M_{\Delta}^{2}}
$$

and

$$
\begin{aligned}
& M_{h}^{2}=\frac{1}{2} \lambda_{H} v^{2}, \\
& M_{1}^{2}=m_{1}^{2}+\frac{1}{2}\left(\lambda_{1 H} v^{2}+\lambda_{12} v_{2}^{2}\right), \\
& M_{2}^{2}=\frac{1}{2} \lambda_{2} v_{2}^{2}, \\
& M_{\Delta}^{2}=m_{\Delta}^{2}+\frac{1}{2}\left[\lambda_{2 \Delta} v_{2}^{2}+\left(\lambda_{H \Delta}+\lambda_{H \Delta}^{\prime}\right) v^{2}\right] .
\end{aligned}
$$

The physical masses of the scalars are approximately given by the M's in Eqs. (9). Here we see the mechanism at work: $\lambda_{12}^{\prime}$ induces a suppression from $v_{2}$ to $v_{1}$, and $\lambda_{A}$ induces a further suppression from $v_{1}$ to $v_{\Delta}$. It is useful to write these quartics in terms of the scalar masses and vevs,

$$
\begin{aligned}
\lambda_{A} & =0.008\left(\frac{M_{\Delta}}{500 \mathrm{GeV}}\right)^{2}\left(\frac{v_{\Delta} / \mathrm{keV}}{v_{1} / \mathrm{MeV}}\right), \\
\lambda_{12}^{\prime} & =0.03 \frac{\left(M_{1} / 100 \mathrm{GeV}\right)^{2}\left(v_{1} / \mathrm{MeV}\right)}{\left(v_{2} / 10 \mathrm{GeV}\right)^{3}} .
\end{aligned}
$$

These relations do not depend on the number of steps, as long as perturbation theory holds.

\section{SPECTRUM AND MIXING PHENOMENOLOGY}

The scalar spectrum of this 2-step scenario consists of the 4 aforementioned neutral scalars $\left(h, \delta, s_{1}, s_{2}\right)$, singly and doubly charged scalars $\delta^{+}$and $\delta^{++}$, with masses approximately given by $M_{\Delta}$, two massive pseudoscalar degrees of freedom $\left(a_{\delta}, a_{1}\right)$ with masses approximately given by $M_{\Delta}$ and $M_{1}$, and two massless Goldstone bosons. One of the Goldstones is the longitudinal polarization of the $Z$ boson while the other is a massless Majoron, $J$ [25-27]. We will analyze the Majoron phenomenology in the following section.

The mixings among $C P$ even scalars will have important phenomenological impacts (see Table I for a summary). The mixings between $h-s_{2}, \delta-s_{1}$ and $h-s_{1}$ are given by

$$
\begin{aligned}
& \theta_{h 2} \simeq \frac{\lambda_{2 H} v_{2} v}{M_{h}^{2}-M_{2}^{2}} \simeq 0.16 \lambda_{2 H}\left(\frac{v_{2}}{10 \mathrm{GeV}}\right) \beta_{h 2}, \\
& \theta_{\delta 1} \simeq \frac{\lambda_{A}}{2} \frac{v^{2}}{M_{1}^{2}-M_{\Delta}^{2}} \simeq 10^{-3}\left(\frac{v_{\Delta} / \mathrm{keV}}{v_{1} / \mathrm{MeV}}\right) \beta_{1 \delta}, \\
& \theta_{h 1} \simeq \frac{\lambda_{1 H} v_{1} v}{M_{h}^{2}-M_{1}^{2}} \simeq 1.5 \times 10^{-5} \lambda_{1 H}\left(\frac{v_{1}}{\mathrm{Mev}}\right) \beta_{h 1},
\end{aligned}
$$

where $\beta_{a b} \equiv\left(1-M_{b}^{2} / M_{a}^{2}\right)^{-1}$. First, the Higgs mixing with $s_{2}$ could in principle be sizable. Observations of Higgs production and decay modes together with precision electroweak measurements constrain the mixing angle $\alpha$ with a scalar singlet to be about $\sin \theta_{h 2} \lesssim 0.2-0.3$ for a $200-800 \mathrm{GeV}$ singlet mass [28]. If the scalar is much lighter than the Higgs, for instance in the region $1<M_{2}<10 \mathrm{GeV}$, the constraints on the mixing range from $\sin \theta_{h 2} \lesssim 10^{-3}-10^{-1}$ [29]. This Higgs-singlet mixing can lead to very interesting phenomenology, but it is not an exclusive signature of our model. For small values of $v_{2}$, the invisible Higgs decay to a pair of Majorons strongly constrains this mixing, as we will see later.

The mixing between $\delta$ and $s_{1}$ is quite special, as it leads to drastic deviations from usual type II seesaw phenomenology. For $\delta^{++}$, a new decay channel may open up,

TABLE I. Sizable scalar mixings and their phenomenological impact.

\begin{tabular}{ll}
\hline \hline Mixing & \multicolumn{1}{c}{ Phenomenology } \\
\hline$h-s_{2}$ & Higgs observables, direct $s_{2}$ production \\
$\delta-s_{1}$ & New LHC signatures, $s_{1}$ decay modes \\
$h-s_{1}$ & $s_{1}$ decay modes \\
$s_{1}-s_{2}$ & Irrelevant \\
\hline \hline
\end{tabular}




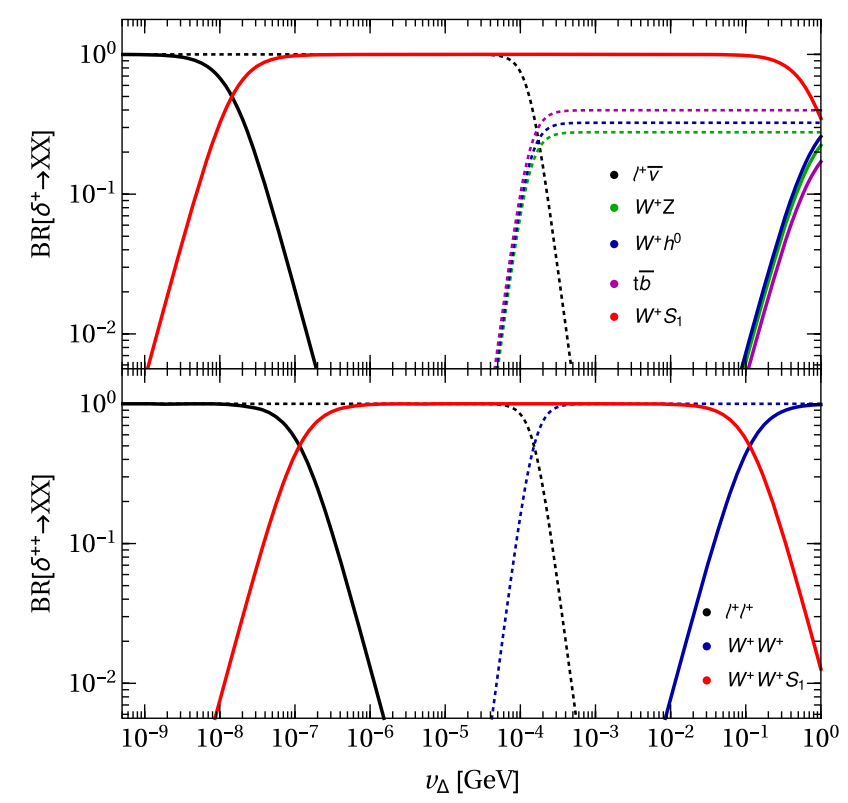

FIG. 2. Branching ratios of $\delta^{+}$(upper panel) and $\delta^{++}$(lower panel) as a function of the triplet vev $v_{\Delta}$ for the usual type II seesaw model (dotted) and our generalized version (solid). We considered $m_{0}=0.1 \mathrm{eV}$, as the lightest neutrino mass, $M_{\delta^{+}}=$ $M_{\delta^{++}}=500 \mathrm{GeV}, M_{1}=100 \mathrm{GeV}$, and $\theta_{\delta 1}=0.005$.

$\delta^{++} \rightarrow W^{+} W^{+} s_{1}$ with $s_{1}$ typically decaying to neutrinos (via mixing with $\delta$ ), quarks or gauge bosons (both via mixing with the Higgs) depending on its mass. Similarly, one can have $\delta^{+} \rightarrow W^{+} s_{1}$ and $\delta \rightarrow h s_{1}$. Another distinctive feature is the possibility of having sizable visible pseudoscalar decays, $a_{\delta} \rightarrow Z s_{1}$. Differently from type II seesaw, these decays are controlled uniquely by gauge coupling and mixing angle $\theta_{\delta 1}$, see Table II. As can be seen in Fig. 2, the new decays can dominate a large region of parameter space in the generalized type II seesaw (solid lines) compared to the usual case (dotted lines). We stress that the mixing angle $\theta_{\delta 1}$ essentially drives the large, novel branching ratio, making the model more predictive. As we will see later, these new decays provide a smoking gun signature at the LHC, not only opening the possibility for discovering new particles, but also distinguishing the model from the type II seesaw.

TABLE II. Typical decay modes in type II seesaw and new modes in the generalized type II framework. In the last column it is indicated the most relevant parameters governing the partial widths.

\begin{tabular}{lccc}
\hline \hline Scalar & Type II & Generalized type II & Parameters \\
\hline$\delta^{++}$ & $\ell^{+} \ell^{+}, W^{+} W^{+}$ & $W^{+} W^{+} s_{1}$ & $v_{\Delta}, \theta_{\delta 1}$ \\
$\delta^{+}$ & $\ell^{+} \nu, W^{+} Z, W^{+} h, t \bar{b}$ & $W^{+} s_{1}$ & $v_{\Delta}, \theta_{\delta 1}$ \\
$\delta$ & $\nu \nu, W^{+} W^{-}, Z Z, h h$ & $h s_{1}$ & $v_{\Delta}, \theta_{\delta 1}$ \\
$a_{\delta}$ & $\nu \nu, t \bar{t}, Z h$ & $Z s_{1}$ & $v_{\Delta}, \theta_{\delta 1}$ \\
$s_{1}$ & not present & $\nu \nu, q \bar{q}, W^{+} W^{-}, Z Z$ & $v_{\Delta}, \theta_{\delta 1}, \theta_{h 1}$ \\
\hline \hline
\end{tabular}

Finally, the mixing between the Higgs and $s_{1}$ given in Eq. (11), although small, plays a significant role in the scalar phenomenology. The $s_{1}$ decay to charged fermions, driven by $\theta_{h 1}$, will compete with the invisible decay to neutrinos, sourced by $\theta_{\delta 1}$. By analyzing the ratio of these partial widths (see Appendix $\mathrm{C}$ for more details),

$$
\frac{\Gamma_{s_{1} \rightarrow \nu \nu}}{\Gamma_{s_{1} \rightarrow f f}} \simeq \frac{3.1}{N_{c}}\left(\frac{\theta_{\delta 1} / 10^{-3}}{\theta_{h 1} / 10^{-5}}\right)^{2}\left(\frac{m_{\nu} / 0.1 \mathrm{eV}}{m_{f} / \mathrm{GeV}}\right)^{2}\left(\frac{\mathrm{keV}}{v_{\Delta}}\right)^{2},
$$

we can see that either visible or invisible $s_{1}$ decays can dominate in large natural regions of the parameter space. In this manuscript we will focus on the latter. Besides, there is some region of parameter space in which $s_{1}$ decays to $b$ quarks and gives rise to displaced vertices at the LHC. We will nevertheless refrain from analyzing that possibility here.

\section{MAJORON PHENOMENOLOGY}

Although a massless particle in the spectrum may at first seem problematic, its couplings to standard model fermions are extremely suppressed due to hierarchy of vevs. The Majoron field is the linear combination

$$
J \simeq \frac{1}{\ell_{2} v_{2}}\left(\ell_{1} v_{1} a_{1}+\ell_{2} v_{2} a_{2}+\frac{1}{2} v_{\Delta} a_{\delta}-\frac{v_{\Delta}^{2}}{v} a\right),
$$

where $\ell_{1}=1$ and $\ell_{2}=1 / 3$ are lepton numbers of the corresponding scalars. It is straightforward to see that the Majoron has very small couplings to charged fermions given by

$$
\begin{aligned}
G_{J f f} & =\frac{y_{f}}{\sqrt{2}} \frac{v_{\Delta}^{2}}{\ell_{2} v_{2} v}=\frac{1.6 \times 10^{-18}}{\ell_{2}} \frac{\left(m_{f} / \mathrm{GeV}\right)\left(v_{\Delta} / \mathrm{keV}\right)^{2}}{\left(v_{2} / 10 \mathrm{GeV}\right)}, \\
G_{J \nu \nu} & =\sqrt{2} y_{\nu} \frac{v_{\Delta}}{\ell_{2} v_{2}}=\frac{5 \times 10^{-12}}{\ell_{2}} \frac{\left(m_{\nu} / 0.1 \mathrm{eV}\right)}{\left(v_{2} / 10 \mathrm{GeV}\right)},
\end{aligned}
$$

easily avoiding constraints from neutrinoless double beta decay with Majoron emission $G_{J \nu \nu}<(0.8-1.6) \times 10^{-5}$ [30], as well as astrophysical bounds $G_{J e e}<4.3 \times 10^{-13}$ [31]. Although a thermalized Majoron would contribute to increase the effective number of relativistic degrees of freedom by $4 / 7$, the tiny coupling in this scenario leads to very little Majoron production in the early universe.

A stringent bound on Higgs- $S_{2}$ mixing comes from Higgs decaying invisibly to a pair of Majorons [32]. It is straightforward to obtain the approximate constraint [33]

$\theta_{h 2}<1.5 \times 10^{-3}\left[\frac{v_{2}}{10 \mathrm{GeV}}\right]\left[\frac{\Gamma_{h}}{4.2 \mathrm{MeV}} \frac{\mathrm{BR}_{h \rightarrow \text { inv }}}{0.22}\right]^{1 / 2}$

where $\Gamma_{h}$ is the Higgs total width and $\mathrm{BR}_{h \rightarrow \text { inv }}$ is its invisible branching ratio. The Higgs total width has only been measured indirectly, via comparison between onshell and off-shell Higgs production, yielding the modeldependent bound $\Gamma_{h}^{\exp }<13 \mathrm{MeV}$ at $95 \%$ C.L. [34]. The 
Higgs invisible branching ratio has been bounded to be below $0.22[35,36]$. This strong bound on $\theta_{h 2}$ could be alleviated by raising $v_{2}$ to the TeV. Complementary constraints (though still not competitive) come from monojets at the LHC [37].

\section{COLLIDER PHENOMENOLOGY}

In this section, we study the collider phenomenology for the generalised type II seesaw model. The leading production channels for this framework remain the same as in the usual type II, i.e., the charged Higgs states will be dominantly produced in pairs via $s$-channel electroweak boson exchange, leading primarily to associated production of double and single charged Higgs bosons $\delta^{ \pm \pm} \delta^{\mp}$, followed by double charged Higgs pair production $\delta^{++} \delta^{--}{ }^{1}$ Although these two production channels do not present differences in rate between the standard type II seesaw and our new model construction, their corresponding decays display new relevant phenomenological signatures. The $\delta-s_{1}$ mixing engenders new interaction terms from the triplet kinetic term

$$
\mathcal{L} \supset \operatorname{Tr}\left[\left(D_{\mu} \Delta\right)^{\dagger} D_{\mu} \Delta\right],
$$

making the decays $\delta^{ \pm \pm} \rightarrow W^{ \pm} W^{ \pm} s_{1}$ and $\delta^{ \pm} \rightarrow W^{ \pm} s_{1}$ available. Note that these partial widths do not present any $v_{\Delta}$ suppression, instead they depend only on gauge couplings, being equally large in a wide range of parameter space $v_{\Delta} \sim 10^{-7}-10^{-1} \mathrm{GeV}$, distinctly from the usual type II, see Fig. 2.

Therefore, the $p p \rightarrow \delta^{ \pm \pm} \delta^{\mp}$ production channel not only reveals the triplet structure nature of $\delta^{ \pm \pm}$and $\delta^{ \pm}[13,14]$, but can also differentiate our construction from the usual type II model. To explore this phenomenology, we analyse the $p p \rightarrow \delta^{ \pm \pm} \delta^{\mp}$ production at the $\sqrt{s}=13 \mathrm{TeV}$ LHC, focusing on the trilepton plus missing energy signature, with two same flavor and same sign leptons, $e^{ \pm} e^{ \pm} \mu^{\mp}+\mathscr{E}_{T}$ and $\mu^{ \pm} \mu^{ \pm} e^{\mp}+\mathbb{E}_{T}$. The leptons arise from the $W$-boson decays and relevant extra sources of missing energy follow from the dominant $s_{1}$ decay, $s_{1} \rightarrow \nu \bar{\nu}$.

Our model is implemented in FeynRules [38] and the signal sample is generated with MadGraph5 [39]. A Nextto-leading order QCD K-factor of 1.25 has been applied [40]. To obtain a robust simulation of the background components, that display large fake rates, our simulation follows the recent $13 \mathrm{TeV}$ CMS study [41]. Although CMS targets a heavy neutral Majorana lepton $N$, it presents a set of search regions for the high mass regime $m_{N}>m_{W}$, leading to a more sizable $\mathbb{E}_{T}$, that also applies to our model.

\footnotetext{
${ }^{1}$ We have checked that producing one triplet scalar in association with $s_{1}$ is typically subleading, as it is suppressed by the small mixing $\theta_{\delta 1}$. Thus, these production modes will be disregarded here.
}

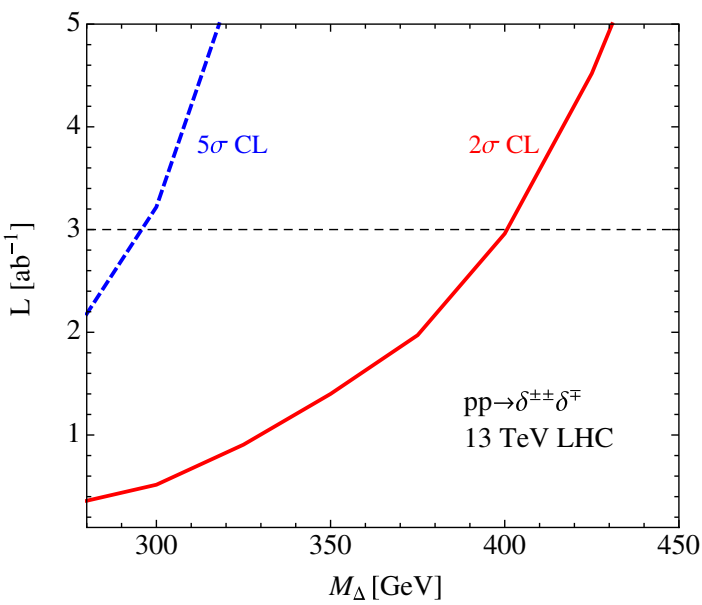

FIG. 3. Luminosity required to observe $p p \rightarrow \delta^{ \pm \pm} \delta^{\mp}$ as a function of $M_{\Delta}$ at $2 \sigma$ (red full) and $5 \sigma$ (blue dashed) confidence level. We assume $M_{1}=100 \mathrm{GeV}$ and $v_{\Delta}=10^{-6} \mathrm{GeV}$.

In this analysis, jets are defined with the anti- $k_{T}$ clustering algorithm with $R=0.4, p_{T j}>25 \mathrm{GeV}$ and $\left|\eta_{j}\right|<2.4$ via FASTJET [42]. Events with one or more $b$-jets are vetoed with $70 \%$ b-tagging efficiency and $1 \%$ mistag rate. Electrons and muons are defined with $\left|\eta_{\ell}\right|<2.4$ and the three leptons must satisfy $p_{T \ell}>55,15,10 \mathrm{GeV}$. Finally, the events are divided in bins associated to three observables: (i) the trilepton mass system $m_{3 \ell}$; (ii) minimum invariant mass of all opposite sign leptons $m_{2 \ell O S^{\prime}}^{\min }$; and (iii) transverse mass $m_{T}=\sqrt{2 p_{T \ell} E_{T}(1-\cos \phi)}$, where $p_{T \ell}$ corresponds to the lepton which is not used in the $m_{2 \ell O S^{\prime}}^{\min }$ calculation and $\phi$ is the azimuthal angle between $\vec{p}_{T \ell}$ and $\vec{p}_{T}^{\text {miss }}$.

Using the CMS background estimate, we perform a binned log-likelihood analysis based on the $\mathrm{CL}_{s}$ method [43], exploring all search regions with $e^{ \pm} e^{ \pm} \mu^{\mp}+\mathbb{E}_{T}$ and $\mu^{ \pm} \mu^{ \pm} e^{\mp}+E_{T}$ displayed by Ref. [41]. In Fig. 3, we present the luminosity required to observe $p p \rightarrow \delta^{ \pm \pm} \delta^{\mp}$ as a function of $M_{\Delta}$ at $2 \sigma$ and $5 \sigma$ confidence level. At the high-luminosity LHC, $\mathcal{L}=3 \mathrm{ab}^{-1}$, we can discover charged Higgses at $5 \sigma$ level up to $M_{\Delta}=300 \mathrm{GeV}$ and exclude them at $2 \sigma$ level up to $M_{\Delta}=400 \mathrm{GeV}$.

A final comment is in order regarding two phenomenological aspects beyond the ones discussed so far. First, our model may also induce lepton flavor violation processes, very similar to the usual type II seesaw scenario [44]. Second, although the model does not have enough $C P$ violation, adding a second $S U(2)$ triplet scalar [45] may lead to successful leptogenesis. The study of such possibilities is beyond the scope of this manuscript.

\section{CONCLUSIONS}

In this paper we have proposed a generalization of type II seesaw in which lepton number is broken dynamically and no hierarchy problem neither arbitrarily small parameters are present. The rich phenomenology of the model includes 
deviations of standard Higgs couplings, the presence of a massless neutral pseudoscalar and more importantly a novel smoking gun signature at the LHC. This distinctive new signature may reveal the triplet nature of the charged scalars and at the same time disentangle the framework from the usual type II seesaw model.

\section{ACKNOWLEDGMENTS}

We thank K. Agashe, E. Bertuzzo and R. Zukanovich Funchal for useful discussions. We are grateful to K. Babu for careful reading of the manuscript. Fermilab is operated by Fermi Research Alliance, LLC, under Contract No. DEAC02-07CH11359 with the U.S. Department of Energy. J. G. and P. A. N.M. acknowledge support from the EU Grants No. H2020-MSCA-ITN-2015/674896-Elusives and No. H2020-MSCA-2015-690575-InvisiblesPlus. D. G. was funded by U.S. National Science Foundation under the Grant No. PHY-1519175. Y. F. P. acknowledges support from Fundação de Amparo à Pesquisa do Estado de São Paulo (FAPESP), under Processes No. 2013/03132-5 and No. 2017/19765-8, and from Conselho Nacional de Desenvolvimento Científico e Tecnológico (CNPq).

\section{APPENDIX A: $\boldsymbol{n}$-STEP GENERALIZED TYPE II SEESAW}

Here we present the generalization of our framework for an arbitrary number of scalar singlets $n$. We define the following scalar bilinears,

$B_{i} \equiv S_{i}^{*} S_{i}, \quad B_{\Delta} \equiv \operatorname{Tr}\left(\Delta^{\dagger} \Delta\right), \quad B_{H} \equiv H^{\dagger} H$,

which allow to write the scalar potential in a compact form

$$
\begin{aligned}
V= & -\frac{m_{H}^{2}}{2} B_{H}+\sum_{\varphi}^{\Delta, 1 \ldots n-1} m_{\varphi}^{2} B_{\varphi}-\frac{m_{n}^{2}}{2} B_{n}+\sum_{\varphi}^{\text {all }} \frac{\lambda_{\varphi}}{4} B_{\varphi}^{2} \\
& +\sum_{\varphi, \varphi^{\prime}>\varphi}^{\text {all }} \lambda_{\varphi \varphi^{\prime}} B_{\varphi} B_{\varphi^{\prime}}+\frac{\lambda_{\Delta}^{\prime}}{4} \operatorname{Tr}\left(\Delta^{\dagger} \Delta \Delta^{\dagger} \Delta\right)+\lambda_{H \Delta}^{\prime} H^{\dagger} \Delta \Delta^{\dagger} H \\
& +\left[\lambda_{A} H^{T} i \sigma_{2} \Delta^{\dagger} H S_{1}^{*}-\frac{2}{3} \sum_{i=1}^{n-1} \lambda_{i, i+1}^{\prime} S_{i}^{*} S_{i+1}^{3}+\text { H.c. }\right] .
\end{aligned}
$$

The notation in the sum of the first term of the second line indicates that permutations of $\lambda_{\varphi \varphi^{\prime}}$ should not be taken (to avoid double counting). Without loss of generality, all $\lambda_{i, i+1}^{\prime}$ and $\lambda_{A}$ can be made real by rephasing the scalar singlet fields. The masses and vevs in the $n$-step realization are approximately given by

$$
\begin{aligned}
& m_{H}^{2}=\frac{1}{2} \lambda_{H} v^{2}+\lambda_{n H} v_{n}^{2}, \\
& m_{n}^{2}=\frac{1}{2} \lambda_{n} v_{n}^{2}+\lambda_{n H} v^{2},
\end{aligned}
$$

$$
\begin{aligned}
& v_{i}=\frac{\lambda_{i, i+1}^{\prime} v_{i+1}^{3}}{3 M_{i}^{2}}, \quad \text { for } i=1, \ldots, n-1, \\
& v_{\Delta}=\frac{\lambda_{A} v^{2} v_{1}}{2 M_{\Delta}^{2}}, \\
& M_{h}^{2}=\frac{1}{2} \lambda_{H} v^{2}, \\
& M_{i}^{2}=m_{i}^{2}+\frac{1}{2}\left(\lambda_{i H} v^{2}+\lambda_{\mathrm{in}} v_{n}^{2}\right), \quad i=1, \ldots, n-1, \\
& M_{n}^{2}=\frac{1}{2} \lambda_{n} v_{n}^{2}, \\
& M_{\Delta}^{2}=m_{\Delta}^{2}+\frac{1}{2}\left[\lambda_{n \Delta} v_{n}^{2}+\left(\lambda_{H \Delta}+\lambda_{H \Delta}^{\prime}\right) v^{2}\right] .
\end{aligned}
$$

These expressions should hold in the regime, $v_{i} \ll v_{i+1}$, that is,

$$
\varepsilon \equiv \lambda_{i, i+1}^{\prime} \frac{v_{i+1}^{2}}{3 M_{i}^{2}} \ll 1
$$

In fact, it is straightforward to show that as long as Eq. (A4) is satisfied, for any number $n$ of scalar singlet fields, the vev of $s_{j}, j<n$, is simply given by

$$
v_{j}=\prod_{k=0}^{n-j-1}\left(\frac{\lambda_{j+k, j+k+1}^{\prime}}{3} \frac{v_{n}^{2}}{M_{j+k}^{2}}\right)^{3^{k}} v_{n} .
$$

If, for simplicity, one takes all $\lambda_{i j}^{\prime}=\lambda^{\prime}$ and $M_{i}=M$, then we obtain a simplified expression,

$$
v_{j}=\left(\frac{\lambda^{\prime}}{3} \frac{v_{n}^{2}}{M^{2}}\right)^{K} v_{n}, \quad K=\left(3^{n-j}-1\right) / 2 .
$$

We can clearly identify the parametric suppression $\varepsilon^{K}$ responsible for making $v_{1} \ll v_{n}$. For instance, if $\varepsilon=0.01$ and $n=3$ we obtain $v_{1} \sim 10^{-8} v_{n}$. Note that the expressions for the mixing angles defined in Eqs. (11) are valid for any $n$, and thus the phenomenological considerations regarding Higgs couplings, Majoron physics and LHC signatures will still apply.

\section{APPENDIX B: STABILITY OF THE SCALAR POTENTIAL}

Although a complete study on the stability of the scalar potential are not the main focus of this Letter, we provide here sufficient conditions for the stability. The key point is that the quartic couplings $\lambda_{A}$ and $\lambda_{12}^{\prime}$ (or any $\lambda_{i, i+1}^{\prime}$ in the $n$-step scenario) can always yield negative contributions to the potential when the values of the fields go to infinity, independently of their sign. As these couplings are the core 
of the generalized type II seesaw mechanism, it is important to understand how to control these contribution so that the potential is bounded from below. Although a full analysis of the stability would be very complicated, specially in the $n$-step scenario, we can still derive useful sufficient conditions to have stability. The idea is to split the scalar potential into pieces that will isolate each $\lambda_{12}^{\prime}$ or $\lambda_{A}$,

$$
V=V_{A}+V_{12}+\cdots+V_{0}
$$

and require each piece to be independently positive. For now we will focus on $n=2$-steps and generalize the method in the end.

The first piece deals with $\lambda_{A}$. We define

$$
\begin{aligned}
V_{A} \equiv & \lambda_{1 H}\left(S_{1}^{*} S_{1}\right)\left(H^{\dagger} H\right)+\lambda_{1 \Delta}\left\langle\Delta^{\dagger} \Delta\right\rangle\left(S_{1}^{*} S_{1}\right) \\
& +\lambda_{H \Delta}\left(H^{\dagger} H\right)\left\langle\Delta^{\dagger} \Delta\right\rangle+\left(\lambda_{A} H^{T} i \sigma_{2} \Delta^{\dagger} H S_{1}^{*}+\text { H.c. }\right)
\end{aligned}
$$

and require it to be positive. By performing an $S U(2)$ rotation on the field one can always write [46]

$$
i \sigma_{2} \Delta=\left(\begin{array}{cc}
a & 0 \\
0 & b e^{i \alpha}
\end{array}\right), \quad H=\left(\begin{array}{c}
c e^{i \beta} \\
d e^{i \gamma}
\end{array}\right),
$$

and $S_{i}=R_{i} e^{i \phi_{i}}$. Then, it is straightforward to obtain

$$
\begin{array}{ll}
\lambda_{H \Delta}>0, & \lambda_{1 \Delta}>0, \\
\lambda_{1 H}>0, & \left|\lambda_{A}\right|^{2}<\lambda_{1 H} \lambda_{H \Delta} .
\end{array}
$$

Now, we handle $\lambda_{12}^{\prime}$ by defining

$$
\begin{aligned}
V_{12} \equiv & \frac{\lambda_{2}}{4}\left(S_{2}^{*} S_{2}\right)^{2}+\lambda_{12}\left(S_{1}^{*} S_{1}\right)\left(S_{2}^{*} S_{2}\right) \\
& -\left(\frac{2}{3} \lambda_{12}^{\prime} S_{1}^{*} S_{2}^{3}+\text { H.c. }\right),
\end{aligned}
$$

and requiring $V_{12}>0$. This yields

$$
\lambda_{12}>0, \quad \lambda_{2}>0, \quad\left|\lambda_{12}^{\prime}\right|^{2}<\frac{9}{16} \lambda_{12} \lambda_{2} .
$$

We still have to deal with seven quartic couplings. First note that $\lambda_{1}, \lambda_{2 H}$, and $\lambda_{2 \Delta}$ need to be positive, as there is no other quartic left that can compensate for a negative contribution to the potential sourced by these couplings. The remaining parameters, $\lambda_{\Delta}, \lambda_{\Delta}^{\prime}, \lambda_{H \Delta}$ and $\lambda_{H \Delta}^{\prime}$, essentially define a usual type II seesaw potential and the stability conditions for that case are known [46]. The requirements for these seven quartics can be summarized as

$$
\text { (i) } \lambda_{H}>0, \quad \lambda_{1}>0, \quad \lambda_{2 H}>0, \quad \lambda_{2 \Delta}>0,
$$

(ii) $\lambda_{\Delta}+\lambda_{\Delta}^{\prime}>0, \quad 2 \lambda_{\Delta}+\lambda_{\Delta}^{\prime}>0$,

(iii) $2 \lambda_{H \Delta}^{\prime}+\sqrt{\lambda_{H}\left(\lambda_{\Delta}+\lambda_{\Delta}^{\prime}\right)}>0$,

$$
\text { (iv) } 2 \lambda_{H \Delta}^{\prime} \sqrt{\lambda_{\Delta}+\lambda_{\Delta}^{\prime}}+\left(2 \lambda_{\Delta}+\lambda_{\Delta}^{\prime}\right) \sqrt{\lambda_{H}}>0 .
$$

We emphasize that if inequalities (B4), (B6), and (B7) are all satisfied, then the potential is stable.

The generalization to more $n$-steps is now straightforward. By defining

$$
\begin{aligned}
V_{i, i+1} \equiv & \frac{\lambda_{i+1}}{4}\left(S_{i+1}^{*} S_{i+1}\right)^{2}+\lambda_{i, i+1}\left(S_{i}^{*} S_{i}\right)\left(S_{i+1}^{*} S_{i+1}\right) \\
& -\left(\frac{2}{3} \lambda_{i, i+1}^{\prime} S_{i}^{*} S_{i+1}^{3}+\text { H.c. }\right)
\end{aligned}
$$

and requiring $V_{i, i+1}>0$ we obtain

$$
\lambda_{i, i+1}>0, \quad \lambda_{i+1}>0, \quad\left|\lambda_{i, i+1}^{\prime}\right|^{2}<\frac{9}{16} \lambda_{i, i+1} \lambda_{i+1}
$$

for $i=1 \ldots n-1$. Again, there are no quartic couplings left to compensate for $\lambda_{i H}$ or $\lambda_{i \Delta}$, which demands

$\lambda_{i}>0, \quad \lambda_{i H}>0, \quad \lambda_{i \Delta}>0, \quad i=1 \ldots n$.

These conditions are by no means necessary, but only sufficient for having stability in the $n$-step realization. More general conditions may be obtained with the techniques of Ref. [47].

\section{APPENDIX C: PARTIAL WIDTHS}

We present in this Appendix the partial widths for the novel decay channels of some of the extra scalars in the generalized type II seesaw framework. In the case of $\delta$, we will have three new channels: $\delta \rightarrow h s_{1}, \delta \rightarrow h h s_{1}$, and $\delta \rightarrow h s_{1} s_{1}$. As the latter is suppressed by $v_{1}^{2}$, we will safely neglect it in the remainder. The partial widths for the first two channels are

$$
\Gamma\left(\delta \rightarrow h s_{1}\right) \simeq \frac{v^{2}}{1024 \pi M_{\Delta}}\left(8 \lambda_{A} \cos \left(2 \theta_{\delta 1}\right)-\lambda_{1 H} \sin \left(2 \theta_{\delta 1}\right)\right)^{2},
$$

$\Gamma\left(\delta \rightarrow h h s_{1}\right) \simeq \frac{M_{\Delta}}{8192 \pi^{3}}\left(2 \lambda_{A} \cos \left(2 \theta_{\delta 1}\right)-\lambda_{1 H} \sin \left(2 \theta_{\delta 1}\right)\right)^{2}$,

where we have neglected the phase space factor by assuming $M_{1}+2 M_{h} \ll M_{\Delta}$. The phase space for 2-body decay can easily be incorporated by multiplying the partial width by

$$
\bar{\beta}_{\delta \rightarrow h s_{1}} \equiv \sqrt{1-\frac{2\left(M_{1}^{2}+M_{h}^{2}\right)}{M_{\Delta}^{2}}+\frac{\left(M_{1}^{2}-M_{h}^{2}\right)^{2}}{M_{\Delta}^{4}}} .
$$


The decay width ratios with respect to the leptonic channel, $\delta \rightarrow \nu \nu+\bar{\nu} \bar{\nu}$, are approximately given by

$$
\begin{aligned}
& \frac{\Gamma\left[\delta \rightarrow h s_{1}\right]}{\Gamma[\delta \rightarrow \nu \nu+\bar{\nu} \bar{\nu}]} \simeq \lambda_{A}^{2} \frac{v_{\Delta}^{2}}{\sum_{i} m_{\nu_{i}}^{2}} \frac{v^{2}}{M_{\delta}^{2}}, \\
& \frac{\Gamma\left[\delta \rightarrow h h s_{1}\right]}{\Gamma[\delta \rightarrow \nu \nu+\bar{\nu} \bar{\nu}]} \simeq \frac{\lambda_{A}^{2}}{512 \pi^{2}} \frac{v_{\Delta}^{2}}{\sum_{i} m_{\nu_{i}}^{2}} .
\end{aligned}
$$

In the case of the single-charged scalar $\delta^{+}$, the additional channel $\delta^{+} \rightarrow W^{+} s_{1}$ is the most relevant. Its decay width is given by

$$
\Gamma\left[\delta^{+} \rightarrow W^{+} s_{1}\right]=\cos ^{2} \eta \frac{\sin ^{2}\left(\theta_{\delta 1}\right)}{8 \pi} \frac{M_{\delta^{+}}^{3}}{v^{2}} \bar{\beta}_{\delta^{+}}^{3},
$$

with

$$
\begin{aligned}
\cos ^{2} \eta & \equiv 1-\frac{2 v_{\Delta}^{2}}{v^{2}}, \\
\bar{\beta}_{\delta^{+}} & \equiv \sqrt{1-\frac{2\left(M_{1}^{2}+M_{W}^{2}\right)}{M_{\delta^{+}}^{2}}+\frac{\left(M_{1}^{2}-M_{W}^{2}\right)^{2}}{M_{\delta^{+}}^{4}}} .
\end{aligned}
$$

[1] T. Kajita, Rev. Mod. Phys. 88, 030501 (2016).

[2] A. B. McDonald, Rev. Mod. Phys. 88, 030502 (2016).

[3] P. Minkowski, Phys. Lett. 67B, 421 (1977).

[4] R. N. Mohapatra and J. W. F. Valle, Phys. Rev. D 34, 1642 (1986).

[5] M. Gell-Mann, P. Ramond, and R. Slansky, Conf. Proc. C790927, 315 (1979).

[6] R. N. Mohapatra and G. Senjanovic, Phys. Rev. Lett. 44, 912 (1980).

[7] R. N. Mohapatra and G. Senjanovic, Phys. Rev. D 23, 165 (1981).

[8] T. Yanagida, Conf. Proc. C7902131, 95 (1979).

[9] J. Schechter and J. W. F. Valle, Phys. Rev. D 22, 2227 (1980).

[10] G. Lazarides, Q. Shafi, and C. Wetterich, Nucl. Phys. B181, 287 (1981).

[11] F. Vissani, Phys. Rev. D 57, 7027 (1998).

[12] G. 't Hooft, NATO Sci. Ser. B 59, 135 (1980).

[13] P. Fileviez Perez, T. Han, G.-y. Huang, T. Li, and K. Wang, Phys. Rev. D 78, 015018 (2008).

[14] P. Fileviez Perez, T. Han, G.-Y. Huang, T. Li, and K. Wang, Phys. Rev. D 78, 071301 (2008).

[15] S. Weinberg, Phys. Rev. Lett. 43, 1566 (1979).

[16] E. Dudas and C. A. Savoy, Acta Phys. Pol. B 33, 2547 (2002).

[17] Z.-z. Xing and S. Zhou, Phys. Lett. B 679, 249 (2009).

[18] C. Bonilla, J. C. Romao, and J. W. F. Valle, New J. Phys. 18, 033033 (2016).
The ratio with the leptonic channel is approximately

$$
\frac{\Gamma\left[\delta^{+} \rightarrow W^{+} s_{1}\right]}{\Gamma\left[\delta^{+} \rightarrow \ell^{+} \nu\right]} \simeq 2 \sin ^{2}\left(\theta_{\delta 1}\right) \frac{v_{\Delta}^{2}}{m_{\nu_{i}}^{2}} \frac{M_{\delta^{+}}^{2}}{v^{2}}
$$

For $s_{1}$, we have the decay into charged fermions and neutrinos

$$
\begin{gathered}
\Gamma\left[s_{1} \rightarrow f \bar{f}\right]=N_{c} \frac{M_{1}}{8 \pi} \frac{m_{f}^{2}}{v^{2}} \sin ^{2} \theta_{h 1} \bar{\beta}_{1}, \\
\Gamma\left[s_{1} \rightarrow \nu \nu\right]=\frac{M_{1}}{16 \pi} \frac{m_{\nu}^{2}}{v_{\Delta}^{2}} \sin ^{2} \theta_{\delta 1},
\end{gathered}
$$

where $N_{c}$ is the number of colors and $\bar{\beta}_{1} \equiv\left(1-4 m_{f}^{2} /\right.$ $\left.M_{1}^{2}\right)^{3 / 2}$. We do not present the analytic expressions for the new 3-body decay channel $\delta^{++} \rightarrow W^{+} W^{+} s_{1}$, as it is not particularly illuminating.

[19] H. Ishida, S. Matsuzaki, S. Okawa, and Y. Omura, Phys. Rev. D 95, 075033 (2017).

[20] P.-H. Gu and R. N. Mohapatra, Phys. Rev. D 97, 075014 (2018).

[21] K. Choi, H. Kim, and S. Yun, Phys. Rev. D 90, 023545 (2014).

[22] K. Choi and S. H. Im, J. High Energy Phys. 01 (2016) 149.

[23] D. E. Kaplan and R. Rattazzi, Phys. Rev. D 93, 085007 (2016).

[24] G. F. Giudice and M. McCullough, J. High Energy Phys. 02 (2017) 036.

[25] Y. Chikashige, R. N. Mohapatra, and R. D. Peccei, Phys. Lett. 98B, 265 (1981).

[26] Y. Chikashige, R. N. Mohapatra, and R. D. Peccei, Phys. Rev. Lett. 45, 1926 (1980).

[27] J. Schechter and J. W. F. Valle, Phys. Rev. D 25, 774 (1982).

[28] T. Robens and T. Stefaniak, Eur. Phys. J. C 76, 268 (2016).

[29] J. D. Clarke, R. Foot, and R. R. Volkas, J. High Energy Phys. 02 (2014) 123.

[30] A. Gando et al. (KamLAND-Zen Collaboration), Phys. Rev. C 86, 021601 (2012).

[31] C. Patrignani et al. (Particle Data Group), Chin. Phys. C 40, 100001 (2016).

[32] R. E. Shrock and M. Suzuki, Phys. Lett. 110B, 250 (1982).

[33] B. A. Dobrescu and K. T. Matchev, J. High Energy Phys. 09 (2000) 031.

[34] V. Khachatryan et al. (CMS Collaboration), J. High Energy Phys. 09 (2016) 051. 
[35] (CMS Collaboration)Combined measurements of the Higgs boson's couplings at $\sqrt{s}=13 \mathrm{TeV}$, CERN Technical Report No. CMS-PAS-HIG-17-031, 2018.

[36] G. Aad et al. (ATLAS Collaboration), J. High Energy Phys. 11 (2015) 206.

[37] P. S. B. Dev, R. N. Mohapatra, and Y. Zhang, J. High Energy Phys. 03 (2018) 122.

[38] A. Alloul, N. D. Christensen, C. Degrande, C. Duhr, and B. Fuks, Comput. Phys. Commun. 185, 2250 (2014).

[39] J. Alwall, R. Frederix, S. Frixione, V. Hirschi, F. Maltoni, O. Mattelaer, H. S. Shao, T. Stelzer, P. Torrielli, and M. Zaro, J. High Energy Phys. 07 (2014) 079.

[40] A. G. Akeroyd and M. Aoki, Phys. Rev. D 72, 035011 (2005).
[41] A. M. Sirunyan et al. (CMS Collaboration), Phys. Rev. Lett. 120, 221801 (2018).

[42] M. Cacciari, G. P. Salam, and G. Soyez, Eur. Phys. J. C 72, 1896 (2012).

[43] A. L. Read, J. Phys. G 28, 2693 (2002).

[44] D. N. Dinh, A. Ibarra, E. Molinaro, and S. T. Petcov, J. High Energy Phys. 08 (2012) 125; 09 (2013) 023(E).

[45] E. Ma and U. Sarkar, Phys. Rev. Lett. 80, 5716 (1998).

[46] K. S. Babu, I. Gogoladze, and S. Khan, Phys. Rev. D 95, 095013 (2017).

[47] K. Kannike, Eur. Phys. J. C 76, 324 (2016); 78, 355(E) (2018). 